ISSN 1870-4069

\title{
Un algoritmo multi-objetivo NSGA-II para la programación de los pasos de laminación en caliente de aceros
}

\author{
Carlos A. Hernández ${ }^{1}$, Guadalupe Castilla ${ }^{2}$, Alejandro López ${ }^{1}$, \\ Juana E. Mancilla ${ }^{1}$ \\ 1 Instituto Politécnico Nacional, \\ Escuela Superior de Ingeniería Mecánica y Eléctrica Azcapotzalco, Ciudad de México, \\ México \\ 2 Instituto Tecnológico de Ciudad Madero, Tamaulipas, \\ México \\ cahernandezc@ipn.mx,gpe_cas@yahoo.com.mx
}

\begin{abstract}
Resumen. El problema de la programación de los pasos de laminación en caliente de aceros es muy complejo, ya que posee grandes espacios de búsqueda de soluciones. El objetivo de este trabajo fue la optimización multiobjetivo del proceso de laminación en caliente de láminas de acero en un tren acabador de seis pasos, implementando el algoritmo genético de búsqueda no dominada elitista de segunda generación (NSGA-II en inglés de Nondominated Sorting Genetic Algorithm), con dos cruzas diferentes (AG1 y AG2) y dos funciones objetivo. Estas fueron el tiempo total de laminado y la flexión de los rodillos de trabajo. Se realizaron dos clases de experimentos computacionales: a) Búsqueda de la mejor solución, b) evaluación comparativa de las configuraciones del NSGA-II. Para cada experimento se obtuvieron 3000 soluciones. En cada molino se calcularon el tiempo de laminación, la fuerza de laminación requerida y los espesores de salida. Para la fuerza se utilizó un modelo constitutivo que calcula la tensión de fluencia, válido para cualquier tipo de acero de bajo, medio carbono y microaleados al Nb, Ti, V. Se reportan las soluciones, seleccionando la mejor de entre 100 con base en el tiempo de cómputo menor. En tiempos menores a 70 s se generaron 100 soluciones. El AG1 tuvo menor tiempo de cómputo que el AG2. Se midió el tiempo de cómputo necesario para generar cada conjunto de soluciones. El AG1 presentó el menor costo computacional. La implementación del NSGA-II minimizó tanto el tiempo de laminación como la flexión de los rodillos de trabajo, a costos computacionales bajos y asegurando que la planicidad de la lámina siempre será menor a la de estándares internacionales.
\end{abstract}

Palabras clave: Algoritmos genéticos, NSGA-II, optimización multiobjetivo, laminación en caliente, programación, pasos de laminación, aceros al carbono. 
Carlos A. Hernández, Guadalupe Castilla, Alejandro López, Juana E. Mancilla

\title{
A NSGA-II Multi-Objective Algorithm for Hot Rolling Pass Schedule of Steels
}

\begin{abstract}
The problem of Scheduling Hot Rolling Pass of Steels is very complex because it has large spaces search for solutions. The aim of this work was the Multi-Objective Optimization (MOO) of hot rolling steel sheets in a finishing train of six steps, implementing the second generation of Nondominated Sorting Genetic Algorithm (NSGA-II). It was used two different crossover (AG1 y AG2) operators and two objective functions. These were the total time of rolling and bending of the work rolls. Two kinds of computational experiments were performed: a) Search for the best solution, b) benchmarking of both NSGA-II configurations. For each experiment was obtained 3000 solutions. Rolling time, separation roll force, and exit thickness for each rolling stand are calculated. A flow stress constitutive model for roll force was used, valid for any low, medium carbon and $\mathrm{Nb}, \mathrm{Ti}, \mathrm{V}$ microalloyed steels. The solutions are reported, choosing some of them between 100 based on the lower computing time. The 100 solutions were generated in times lower than $70 \mathrm{~s}$. AG1 had lower computing times AG2. The NSGA-II implementation minimized both the rolling time as crown of the work rolls, at low computational costs and ensuring that the flatness of strip will always be lower than international standards.
\end{abstract}

Keywords: Genetic algorithms, NSGA-II, multi-objective optimization, hot rolling, schedule, pass rolling, carbon steels.

\section{Introducción}

El proceso de laminación de aceros en caliente es uno de los métodos de conformado más utilizados para la obtención de productos para diferentes industrias metalmecánicas. En este proceso, el espesor de un planchón de acero se reduce gradualmente aplicando fuerzas de comprensión mediante rodillos de laminación [1]. Las fuerzas de laminación aplicadas tienden a incrementarse a medida que el porcentaje de reducción del espesor de la lámina aumenta, pudiendo llegar a provocar flexión en los rodillos de trabajo, pudiendo provocar imperfecciones en la superficie de la lámina o defectos de planicidad por corona de flexión. Esto último produce una lámina que es más delgada en el centro que en las orillas [2]. Existen métodos mecánicos para corregir este tipo de problemas, como el rectificado de los rodillos. Así, cuando el rodillo se somete a fuerzas de separación y se flexiona se obtendrá un espesor constante en la lámina. En el caso de laminación de aceros, el radio del punto máximo de la comba es en general $0.25 \mathrm{~mm}$ mayor que en los extremos de los rodillos [3]. Esto implica que el rodillo sólo puede someterse a una carga específica, lo cual en la industria es ineficiente. De no ser así, las láminas producidas por este rodillo presentarían defectos de 
Un algoritmo multi-objetivo NSGA-II para la programación de los pasos de laminación ...

planicidad. Además, una mala selección de los pasos de laminación incrementa el tiempo del proceso, lo cual es improductivo.

Cuando la solución de un problema tiene un espacio de búsqueda muy amplio, existen limitaciones de tiempo para llegar a una solución útil y no existe un método analítico mediante el cual se pueda llegar a una solución exacta. Por ello se usan técnicas metaheurísticas para encontrar soluciones óptimas alejadas de óptimos locales; es decir, se orienta la búsqueda acorde a la evolución del proceso. Los algoritmos evolutivos utilizan mecanismos inspirados en la evolución biológica, tales como la selección, reproducción y la mutación. Las soluciones se modeladan como individuos de una población. Una función de aptitud determina el entorno donde se encuentran las soluciones. Dentro de los algoritmos evolutivos están los algoritmos genéticos (AG), que son una técnica de búsqueda iterativa, buscando derivar estrategias de optimización basadas en la generación de poblaciones de individuos aptos mediante la reproducción de los padres [4]. Los problemas reales usualmente requieren la búsqueda de soluciones que satisfagan en forma simultánea múltiples criterios de desempeño u objetivos que pueden ser contradictorios, es decir, se desconoce la manera óptima de combinar los diferentes objetivos o que sea inadecuado, cuando no es imposible hacerlo, a lo cual se le conoce como un Problema de Optimización Multiobjetivo (MOP en inglés de Multiobjective Optimization Problem) [5].

Ya que el proceso de laminación en caliente obedece a más de una variable, el espacio de soluciones óptimas puede ser infinito y el tiempo para obtener solo una de ellas con métodos analíticos puede tomar demasiado tiempo y disminuir la eficiencia del proceso. Por ello, la aplicación de técnicas metaheurísticas a este proceso ha sido objeto de varias investigaciones cuyos autores han enfocado su atención en diferentes variables. En este trabajo se trata de encontrar los espesores intermedios óptimos $\left(h_{1}, \ldots, h_{f-1}\right)$ tales que minimicen tanto el tiempo total de laminación como la flexión máxima de los rodillos de trabajo. Para la flexión, esta deberá ser menor a los límites de planicidad establecidos por algunas normas internacionales como la EN 10 051, ASTM A568 o A653, la JIS G-3193 o G-3116 y la DIN 10051. Por tratarse de una investigación de carácter multiobjetivo, este trabajo se integra a las investigaciones cuyos propósitos son la optimización de las variables intrínsecas del proceso, para reducir el tiempo total de laminación, así como la optimización de la operación y vida útil de la maquinaria para disminuir la máxima flexión de los rodillos de trabajo de la cédula de laminación. Por ello, en las últimas dos décadas, el proceso de laminación ha sido objeto de estudio aplicando diferentes técnicas metaheurísticas y, más recientemente, de algoritmos evolutivos para la optimización multiobjetivo del proceso, por el hecho de que existe más de una restricción, algunas de las cuales se mencionan a continuación.

Nolle y Armstrong [3] realizaron la modelización del proceso de un tren acabador de laminación de siete pasos aplicando redes neuronales [6], dirigido a la optimización de factores cuyos parámetros se reflejaban en la planicidad y la deformación longitudinal de una lámina obtenida mediante un tren laminador. Un desarrollo importante en el campo de la aplicación de los algoritmos 
genéticos $(\mathrm{AG})$ a la laminación en frío fue propuesto por Wang y cols. [7] quienes optimizaron la programación de una cédula de laminación en frío. En su investigación obtuvieron los parámetros que optimizaban el proceso infiriendo el incremento en la calidad de la lámina y el aumento de la vida útil de los rodillos de laminación. Wang demostró que los parámetros se mejoraron destacando la reducción de la potencia empleada, las revoluciones por minuto necesarias para el proceso, así como el par requerido. En 2003 Nolle y Zelinka [8] desarrollaron un algoritmo heurístico de optimización denominado algoritmo de migración auto organizado (SOMA del inglés Self-Organizing Migration Algorithm ). Tanto el objetivo de ese trabajo como la función a optimizar fueron los mismos del trabajo publicado en 1999 [3]. SOMA se aplicó en un programa de laminación para catorce diferentes láminas y se relacionó con la calidad de las láminas obtenidas por el proceso de laminación. Los resultados arrojados se compararon con los de la planicidad de láminas medidas en I-Units provenientes de pruebas industriales, obteniendo SOMA un $33 \%$ de mejora. Estos autores también desarrollaron un AG para la optimización mediante simulación de los rodillos de trabajo del tren acabador. El factor a optimizar fue una función compuesta por una combinación de parámetros de planicidad. Se comparó el AG con ocho diferentes algoritmos de optimización experimentales, como lo son el recocido simulado, ascenso de colina y la trayectoria aleatoria. Demostraron que el AG fue el que obtuvo los mejores resultados. En 2007, Nolle [9] implementó un algoritmo heurístico de búsqueda de tamaño de pasos auto adaptable (SASS del inglés self-adaptive step size search), para optimizar el perfil de los rodillos de trabajo utilizados en el proceso de laminación en caliente. El SASS es un algoritmo basado en los principios aplicados en la técnica de optimización experimental de ascenso de colina, donde únicamente se puede configurar el tamaño de la población y aunque el algoritmo es muy simple, los resultados obtenidos fueron muy buenos. Los resultados arrojados por SASS se valoraron en relación con la planicidad de la lámina y su deformación longitudinal, siendo mejores que los datos industriales contra los cuales se compararon. Recientemente, Pholdee et al. [10] optimizó la planicidad de láminas durante el bobinado utilizando un método de evolución diferencial junto con varios algoritmos evolucionarios. Este método realiza gran cantidad de iteraciones, por lo cual requiere nuevos algoritmos evolucionarios que reduzcan la cantidad de evaluaciones a las funciones.

\section{Formulación del problema de optimización}

El problema de la optimización de la programación de los pasos de laminación en caliente para la fabricación de lámina plana radica en que la minimización del tiempo de procesamiento no toma en cuenta el efecto que tendría sobre la flexión del rodillo de trabajo y con ello la afectación del perfil de la lámina, tal que sobrepase las tolerancias cada vez más exigentes para la calidad de las láminas. Ya que las minimizaciones del tiempo de laminación y de la flexión del rodillo involucran soluciones en conflicto se requiere resolver el problema de optimización mediante algoritmos multiobjetivos. 
Existen muchos métodos de solución para encontrar un conjunto de soluciones lo más cercano posible a un frente óptimo de Pareto de los tiempos de laminación $t$ y la flexión del rodillo $\nu$, en cada paso de laminación. Ya que las fuerzas de laminación están gobernadas por los parámetros del proceso y por la resistencia a la deformación del material, conviene que el tiempo de laminación y la flexión del rodillo óptimos provengan de un NSGA-II, ya que permite hallar un número de soluciones no dominadas que convergen en un frente óptimo de Pareto. Para este trabajo se han considerado tres restricciones, a) la potencia óptima de laminación $\left(P_{\text {ópt }}\right)$ para obtener un espesor de salida $\left(h_{i}\right)$ sea menor que la potencia nominal ( $\left.P_{\text {nominal }}\right)$ de cada molino de laminación, b) el espesor de salida $\left(h_{i}\right)$ sea estrictamente menor que el espesor de entrada $\left(h_{i-1}\right)$ y c) la corona de flexión $\left(\nu_{\text {ópt }}\right)$ sea menor a la establecida por las normas de planicidad $\left(\nu_{\text {norma }}\right)$. Entonces el problema de optimización se formula como

$$
\left.\begin{array}{ll}
\text { minimizar } & f_{1}(t, \nu)=\sum_{n=1}^{n} t_{i}, \\
\text { minimizar } & f_{2}(t, \nu)=\frac{R_{A} x^{3}}{6 E I}-\frac{q\left(x-l_{1}\right)^{4}}{24 E I}+\left(\frac{q x\left(\frac{L}{2}-l_{1}\right)^{3}}{6 E I}-\frac{R_{A} x L^{2}}{8 E I}\right), \\
\text { sujeto a: } & P_{\text {ópt }}<P_{\text {nominal }} \\
& h_{i-1}<h_{i} \\
& \nu_{\text {ópt }}<\nu_{\text {norma }},
\end{array}\right\}
$$

siendo $R_{A}$ la reacción en un extremo del rodillo (punto $A$ ), $x$ la distancia longitudinal dada a lo largo de la cual ocurre la flexión del rodillo, $l_{1}$ la longitud de la primera sección del rodillo de trabajo, $L$ la longitud total del rodillo, $E$ el módulo de elasticidad del material del rodillo e $I$ el momento de inercia de la sección transversal del rodillo. Los parámetros de entrada del planchón se pueden consultar en trabajos previos $[11,12]$.

\section{Parámetros del AG}

El algoritmo genético se inicializa mediante parámetros establecidos por el usuario previamente (Tabla 1).

Tabla 1. Parámetros del NSGA-II.

\begin{tabular}{lc}
\hline Parámetro & Magnitud \\
\hline Población inicial & 100 \\
Número de generaciones & 100 \\
Taza de cruzamiento & $0.9 \%$ \\
Taza de mutación & $0.2 \%$ \\
\hline
\end{tabular}




\section{Instancias empleadas}

Las instancias para la experimentación en este trabajo se obtuvieron de trabajos previos [13]. Para determinar la funcionalidad del AG desarrollado, así como sus variantes en el tipo de cruza, se probaron 17 instancias. Cada instancia representa un problema diferente. En cada problema se determinaron las reducciones intermedias para laminar un acero hasta obtener el espesor final deseado en un tren de laminación de 6 pasos. Cada instancia contiene el espesor inicial del planchón $\left(h_{0}\right)$, el espesor final $\left(h_{f}\right)$ deseado, la composición química del material ( \% C, \% Mn, \% Si, \%Mo, \% Nb, \% Ti, \%V) y el ancho del planchón $(w)$. También, y para cada paso del tren de laminación, se deben especificar diámetros de rodillos de trabajo $\left(D_{i}\right)$, velocidades de rodillos $\left(v_{i}\right)$, distancias entre las cajas de laminación $\left(l_{i}\right)$, potencias de motores $\left(P_{i}\right)$, temperaturas intermedias $\left(T_{i}\right)$ y el tamaño inicial de grano $\left(d_{0_{i}}\right)$.

\section{Solución al problema de optimización}

Para dar solución al problema de optimización de una cédula de laminación en caliente en esta investigación se empleó el NSGA-II. Se configuró al AG en dos formas diferentes. En la primera se utilizó la cruza en dos puntos (AG1) y en la segunda se utiliza cruza SBX (AG2). En ambos casos se implementó una selección aleatoria elitista para generar la población inicial y se mantuvo una mutación constante.

La población inicial está compuesta sólo por individuos factibles, que evolucionan en busca de la mejor solución al problema. El número de individuos lo define el usuario al inicio de la ejecución del algoritmo, al igual que el número de generaciones, que representa el ciclo del algoritmo.

\subsection{Algoritmo NSGA-II}

En el Algoritmo 1 se muestra el código básico del NSGA-II. Para que se genere la primera población de padres y cumpla su ciclo de operación, los individuos deben cumplir con todas las restricciones del problema.

En el Algoritmo 2 se muestran las operaciones más importantes, así como los cálculos para determinar las restricciones del problema con las cuales se clasifica a un individuo como viable o no viable.

\subsection{Generación población inicial}

Dado un espesor inicial $h_{0}$ y un espesor final $h_{f}$ del planchón, considerados constantes del problema, se generan individuos cuya representación es la siguiente:

$$
X=\left[h_{0}, h_{1}, h_{2}, h_{3}, h_{4}, h_{5}, h_{f}\right] .
$$

A continuación se explica brevemente la solución obtenida para la instancia número 1. Con los datos de la instancia y mediante la ecuación (3), que representa 
Un algoritmo multi-objetivo NSGA-II para la programación de los pasos de laminación ...

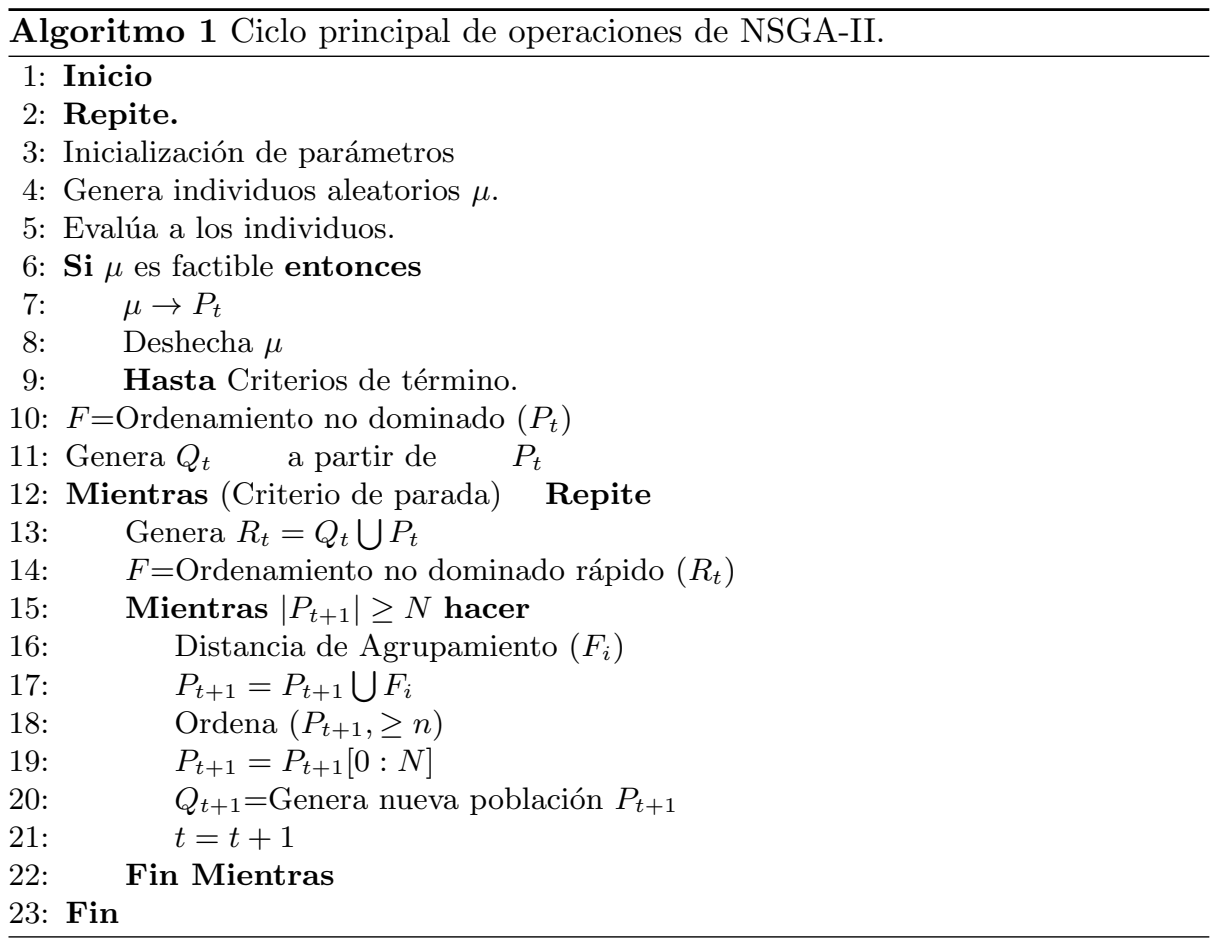

la reducción media $\bar{r}$ para $n$ pasos de laminación $(=6)$, se obtienen los individuos de la población inicial:

$$
\bar{r}=1-\sqrt[n]{\frac{h_{f}}{h_{0}}} .
$$

Cada individuo representa una solución al problema:

$$
X=[48,36,25,18,10,6,38],
$$

e implica que tal individuo es factible al haber cumplido con las restricciones del problema.

La población de 100 individuos generada representa a la población inicial $\left(P_{0}\right)$, que se clasifica según los niveles de no dominancia en frentes de Pareto $\left(F_{1}, F_{2}, \ldots, F_{n}\right)$. Una vez que todos los individuos se han ordenado, se les asignó una calificación denominada aptitud. Los individuos que ocupan el frente $F_{1}$ reciben la mejor calificación, debido a que son individuos no dominados, como se muestra en la Tabla 2.

\subsection{Cruza}

Se considera a cada individuo como la representación de una solución para el problema y cada solución tiene un rango de no dominancia asociado $\left(f_{i}\right)$, así como una distancia de apilamiento $\left(d_{i}\right)$. 


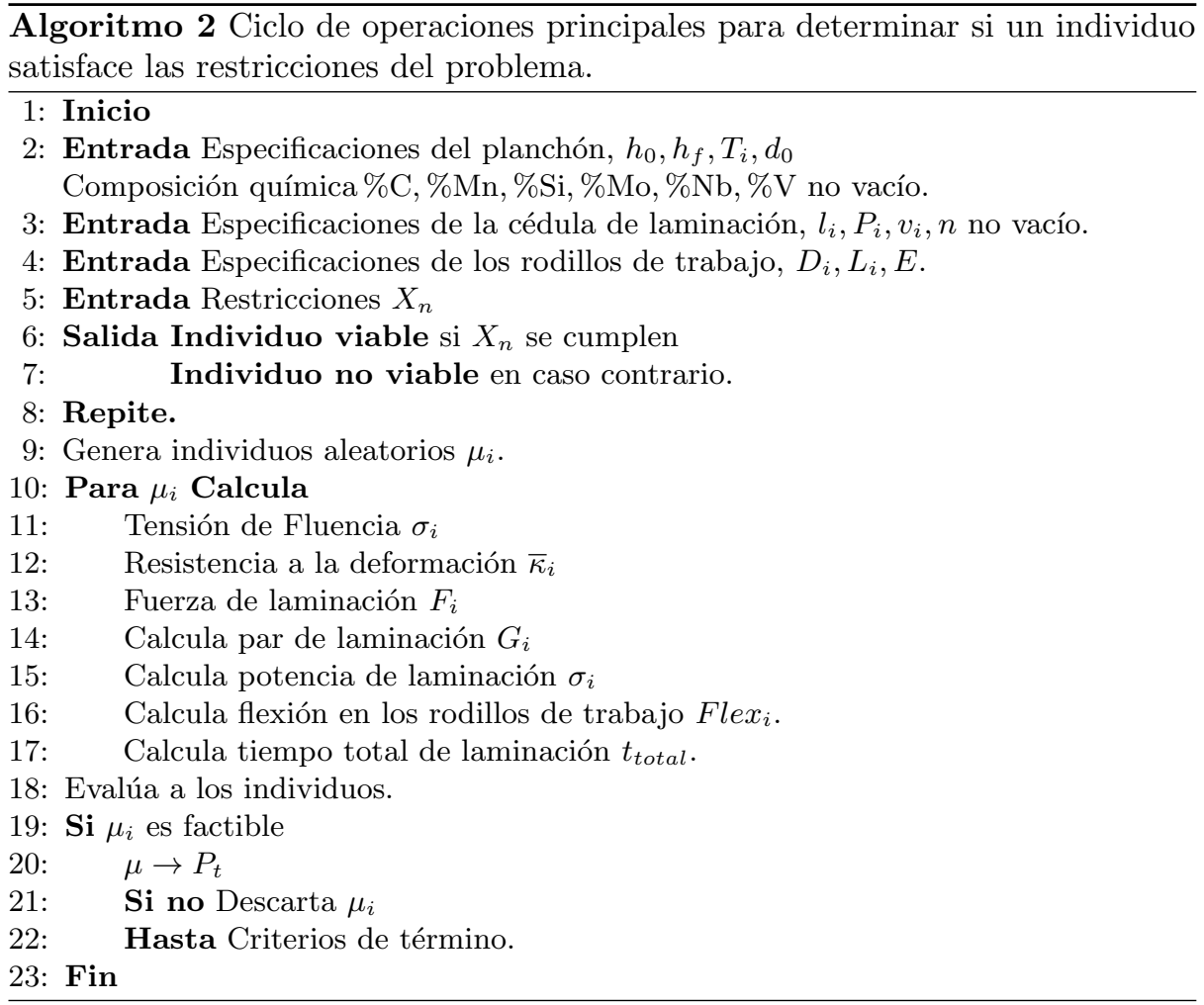

Tabla 2. Ordenamiento en frentes no dominados de Pareto.

\begin{tabular}{ccc}
\hline Frente & Padres & Fitness \\
\hline$F_{1}$ & $2,8,9,5$ & 10 \\
$F_{2}$ & $1,3,4$ & 9 \\
$F_{3}$ & 7,6 & 8 \\
$F_{4}$ & $\cdots$ & 7 \\
$F_{n}$ & $\cdots$ & $\cdots$ \\
& $\sum$ Padres $=100$ & \\
\hline
\end{tabular}

Una vez completado el ordenamiento de la población inicial de padres $P_{0}$ en los frentes de Pareto se procedió a su cruza mediante la técnica de torneo binario. Esta comienza con la selección aleatoria de dos individuos de la población de padres. La mejor solución es por comparación y el ganador es el que tiene la mejor calificación o rango de no dominancia. En el caso de estar situadas en el mismo frente de Pareto, la ganadora del torneo es aquella que tiene mejor distancia de apilamiento, también conocida como distancia de Crowding $d_{i}$, que representa la mayor distancia entre las soluciones de la población asegurando la diversidad de soluciones. 
La $d_{i}$ se calcula mediante la ecuación (4),

$$
d_{I_{j}^{m}}=\frac{f_{m}^{I_{j+1}^{m}}-f_{m}^{I_{j-1}^{m}}}{f_{m}^{\text {máx }}-f_{m}^{\text {mín }}},
$$

siendo $f_{m}^{\text {máx }}, f_{m}^{\text {mín }}$ los valores máximo y mínimo de la función objetivo $m$ y $f_{m}^{I_{j+1}^{m}}, f_{m}^{I_{j-1}^{m}}$ son las soluciones vecinas a la $j$-ésima configuración para cada una de las funciones objetivo $m$. La distancia resultante fue la suma de las distancias en cada una de las direcciones de las funciones objetivo. Se dio preferencia a aquellos individuos localizados en una región menos poblada para generar dispersión en las soluciones.

Una vez que los padres ganadores del torneo binario se seleccionaron, se realizó la cruza entre ellos para generar una población de tamaño $N$. Los métodos de cruza fueron la de dos puntos y SBX. Para el cálculo de la primera generación del hijo ${ }_{1}$, sólo se consideran los $h_{1}$ de ambos padres. Aplicando la ecuación para la cruza $\mathrm{SBX}$, cuando $\beta=1.5, P_{1}=0.032 \mathrm{~m}$ y $P_{2}=0.034 \mathrm{~m}$ se tiene que:

$$
\begin{aligned}
& H_{1}=0.5\left[\left(P_{1}+P_{2}-\beta\left(P_{1}-P_{2}\right)\right]=0.0315\right. \\
& H_{2}=0.5\left[\left(P_{1}+P_{2}+\beta\left(P_{1}-P_{2}\right)\right]=0.0345 .\right.
\end{aligned}
$$

\subsection{Mutación}

El operador mutación es necesario para preservar la diversidad genética de la población evitando divergencia prematura. En este trabajo se implementó mutación uniforme para el desarrollo del NSGA-II. La forma en que se desarrolla este tipo de mutación es la siguiente:

$$
\begin{aligned}
P & =\left[h_{0}, h_{1}, h_{f}\right], \\
P^{\prime} & =\left[h_{0}, h_{1}^{\prime}, h_{f}\right],
\end{aligned}
$$

siendo $h_{1}^{\prime}=$ random $(L I, L S)$. Se utilizó una distribución uniforme y $(L I, L S)$ definen los límites mínimos y máximos de la variable $h_{1}^{\prime}$.

\subsection{Unión padres e hijos}

En este algoritmo, la población descendiente $Q_{t}$ de tamaño $N$ se crea en primera instancia utilizando la población de padres $P_{t}$ de tamaño $N$. Después, las dos poblaciones se combinan para formar $R_{t}$ de tamaño $2 N$. A esta nueva población $R_{t}=P_{t} \bigcup Q_{t}$ se le deberán aplicar todas las operaciones para generar la nueva población de individuos. Sin embargo, como la población $R_{t}$ es de tamaño $2 N$ y solamente existen $N$ configuraciones que conforman la población descendiente, no todas las configuraciones de los frentes pertenecientes a la población $R_{t}$ podrán integrarse a la nueva población, por lo cual aquellos frentes 
que no pueden ser acomodados desaparecen, es decir no son considerados en las nuevas poblaciones, asegurando así que sólo los frentes más aptos sigan reproduciéndose.

El ciclo de operaciones del NSGA-II es repetitivo en todas las generaciones, por lo cual sucesivamente se crea la población descendiente $Q_{i+1}$, a partir de $P_{i+1}$, aplicando selección por torneo para apilamiento, cruza y mutación, hasta que la instrucción de paro del algoritmo se cumpla. La instrucción de paro es parte de los parámetros de entrada del algoritmo.

\section{Evaluación comparativa}

Debido a que se implementó el NSGA-II configurado en dos distintas formas, modificando la técnica de cruza en cada uno de ellos, es conveniente establecer si el tipo de configuración afecta los resultados obtenidos. El objetivo de la evaluación comparativa fue identificar si existen diferencias en los resultados obtenidos de las diferentes configuraciones del NSGA-II.

En la Tabla 3 se muestran las dos configuraciones utilizadas en este trabajo.

Tabla 3. Configuraciones del NSGA-II para este estudio.

\begin{tabular}{cccc}
\hline Algoritmo Tipo de selección & Cruza & Mutación \\
\hline AG1 & E. por torneo & 2 puntos & Uniforme \\
AG2 & E. por torneo & SBX & Uniforme \\
\hline
\end{tabular}

Se consideró al costo computacional para propósitos de la evaluación. Para realmente lograr una comparación objetiva entre soluciones, es necesario definir una medida cuantitativa de la calidad de las mismas. Para problemas de optimización multiobjetivo donde no existe una solución única sino un conjunto de soluciones, no es fácil definir un criterio para determinar cuándo un conjunto es mejor que otro debido a que se espera que un conjunto de soluciones se encuentre compuesto únicamente por soluciones mutuamente no comparables. Es decir, para cualquier par de soluciones $\overrightarrow{x_{1}}, \overrightarrow{x_{2}}$ no ocurre que $\overrightarrow{x_{1}} \leq \overrightarrow{x_{2}}$ ni $\overrightarrow{x_{1}} \geq \overrightarrow{x_{2}}$ debido a que todas ellas son viables y es el usuario quien debe seleccionar una de modo manual o programado.

Es posible definir un indicador de calidad que asigna un valor real a un conjunto de aproximación del frente de Pareto. Un indicador debe definir un orden total en los conjuntos de aproximación del frente de Pareto. Si se tienen dos conjuntos de aproximación $A, B$ tales que $I(A)>I(B)$, entonces el conjunto $A$ es preferible sobre $B$. Se puede asignar un indicador particular. Por tal motivo puede ocurrir que si $I(A)>I(B)$ utilizando algún indicador, entonces $I^{\prime}(A)>$ $I^{\prime}(B)$ tendrá un indicador distinto. 
Un algoritmo multi-objetivo NSGA-II para la programación de los pasos de laminación ...

\section{Condiciones de la experimentación}

Las condiciones fueron las siguientes:

- Se empleó una computadora con procesador Intel Core $i 7$ con una velocidad máxima de procesamiento de $3.1 \mathrm{GHz}$ y 12 GB RAM.

- Se realizaron 30 corridas por cada una de las 17 instancias para obtener los valores promedios de cada una de ellas así como garantizar la fiabilidad de los resultados. Cada corrida contiene en un archivo de salida con 100 soluciones, todas viables porque cumplieron con las restricciones del problema.

Se implementó el algoritmo bajo los parámetros muestrados en la Tabla 4.

Tabla 4. Parámetros del NSGA-II

\begin{tabular}{ll}
\hline Parámetro & Magnitud \\
\hline Población inicial & 100 ind. \\
Núm. generaciones & 100 gen. \\
Cruza & $95 \%$ \\
Mutación & $20 \%$ \\
\hline
\end{tabular}

Para cada experimento se obtuvieron tres mil soluciones que satisfacen a las funciones objetivo de este problema. Además, en cada molino se obtuvo el tiempo de laminación, la fuerza de laminación y los espesores intermedios óptimos. Así mismo se obtuvieron los tiempos de cómputo de cada experimento.

\section{Búsqueda de la mejor solución}

En otras propuestas de algoritmos publicados en la literatura para el problema de laminación en caliente, el espacio de soluciones quedó restringido a la búsqueda de únicamente una solución por cada experimento que entregan buenos resultados, pero suelen tener altos tiempos de cómputo. Las soluciones obtenidas en este trabajo mediante el AG sirvieron para seleccionar la mejor de ellas. Esta depende directamente de los requerimientos del usuario, es decir es él quien selecciona una respuesta del conjunto como la mejor, basado en su experiencia y conocimiento del proceso, con la seguridad de que cualquiera de ellas cumple con las restricciones del problema. En esta investigación se seleccionó aquella corrida que generó el menor tiempo de cómputo, evaluándose 100 soluciones. Se consideraron dos indicadores principales, tiempo de laminación y flexiones máximas en los rodillos de trabajo; se dio un peso específico a cada indicador. El indicador con mayor peso es el tiempo de laminación. Por ser un problema de minimización se seleccionó como mejor solución la que presentó aquellos indicadores cuyos valores son los mínimos. Con base en este criterio se obtuvieron los siguientes resultados. 


\subsection{Tiempo de cómputo}

En la Fig. 1 se muestra el tiempo de cómputo promedio que le tomó a cada una de las configuraciones del algoritmo propuesto en generar una corrida para la instancia 1. En un lapso de 15 a 70 segundos, el AG es capaz de generar conjuntos de 100 soluciones.

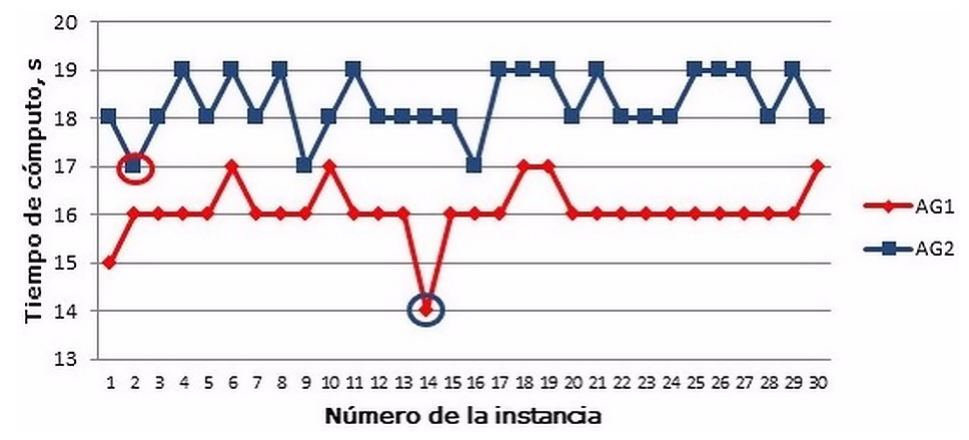

Fig. 1. Tiempo promedio para generar una corrida de la Instancia 1.

Nótese que el AG1 tiene una mejor eficiencia sobre el AG2 pues en todos los casos requiere menor tiempo de cómputo para generar una corrida. Además, para el proceso de selección de la mejor solución de nuestro problema se eligió la corrida que requirió de menor tiempo de cómputo, enmarcadas con circunferencias en dicha Fig. 1. La corrida generada en menor tiempo fue la 14 para el AG1 y la dos para el AG2.

También se seleccionó de entre las 100 soluciones aquella que mejor se adaptó al problema. En la Fig. 2 se muestran los tiempos de laminación para cada una de las 100 soluciones determinadas mediante el AG1, siendo la solución 71 la que tuvo el menor tiempo de laminación.

En la Fig. 3 se muestran los resultados para tiempos de laminación obtenidos mediante el AG2, para la instancia número 1, apreciándose la similitud con los resultados obtenidos mediante el AG1. Sin embargo el tiempo mínimo de laminación obtenido mediante el AG2 es mayor que el del AG1. Se mejoró hasta en un $0.37 \%$ el tiempo de laminación industrial.

\section{Evaluación comparativa entre las diferentes configuraciones del NSGA-II}

Parte fundamental de este trabajo fue determinar el costo computacional de cada una de las variantes del algoritmo propuestas, es decir, el tiempo de cómputo necesario para generar cada conjunto de soluciones. El costo computacional 


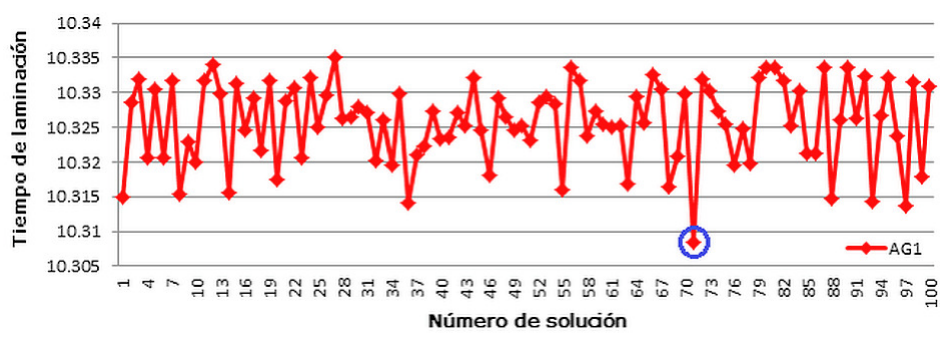

Fig. 2. Tiempos de laminación para la instancia número 1 producidos por el AG1. Con circulo azul la que tuvo el menor tiempo de laminación.

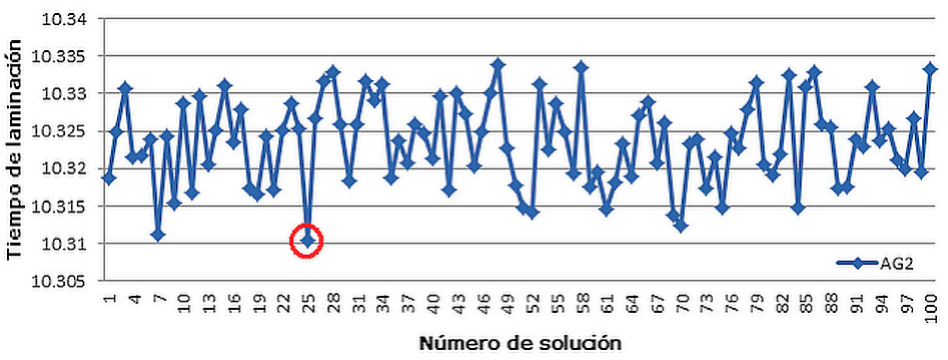

Fig. 3. Tiempos de laminación para la instancia número 1 producidos por el AG2.

se midió desde que inicia la puesta en marcha del algoritmo hasta culminar con la generación del archivo de salida que contiene las soluciones. Ya que el costo computacional es un parámetro utilizado para determinar la mejor solución, fue necesario cronometrarlo.

En la Fig. 4 se muestran los resultados obtenidos aplicando las distintas configuraciones del NSGA-II. Se observa que el AG2, representado en color azul, presentó en todas las instancias mayor costo computacional, es decir tardó mayor tiempo en generar un conjunto de soluciones. Esto se debe en gran medida a que para realizar la cruza SBX es necesario que el algoritmo resuelva ecuaciones para determinar a las nuevas generaciones, a diferencia de la cruza de dos puntos, que emplea un sistema de generación de individuos menos complejo. La diferencia entre los tiempos de cómputo obtenidos en cada una de las corridas aplicando ambas configuraciones del NSGA-II fue muy pequeña, así como en la calidad de la solución obtenida.

En la Fig. 5 se muestra el frente de Pareto con las dos funciones objetivo para el molino 6. Nótese que en la flexión que sufren los rodillos cuando se aplican los parámetros de una cédula de laminación industrial superó los límites establecidos de flexión bajo las normas de planicidad. Por otra parte, las soluciones en el 


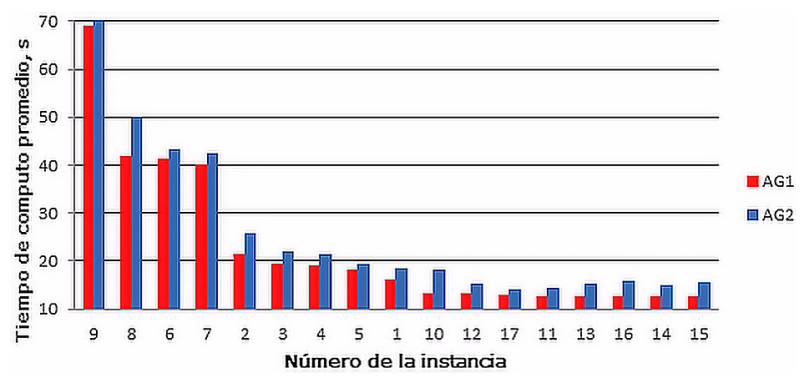

Fig. 4. Comparación entre los tiempos que tarda el AG1 y el AG2 en determinar un conjunto de 100 soluciones.

frente de Pareto encontradas mediante el AG propuesto en todas las ocasiones se mantienen bajo las tolerancias permisibles.

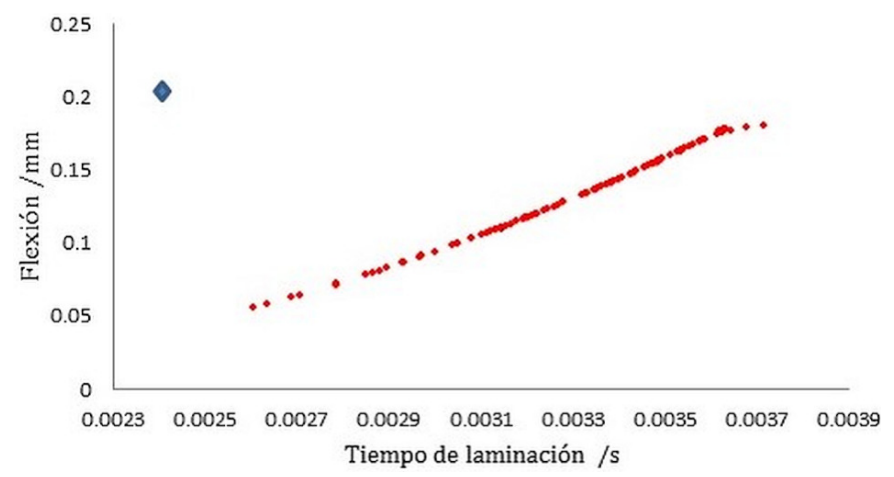

Fig. 5. Frente de Pareto para las flexiones y tiempos calculados mediante el AG (círculos rojos) en comparación con datos industriales (rombo azul).

En la Fig. 6 se muestran las reducciones determinadas por el algoritmo implementado, comparadas con las reducciones reportadas en la cédula de laminación industrial. Se puede decir que las reducciones determinadas mediante el algoritmo están mejor distribuidas en los pasos de laminación, para generar reducciones menos críticas que disminuyan la flexión en los rodillos y la potencia de laminación necesaria. Asimismo, se puede observar que los primeros tres pasos de laminación reducen a más de dos terceras partes el espesor inicial del planchón y los últimos tres pasos son más suaves, ya a que los pasos finales son pasos para dar el acabado que requiere la lámina. 


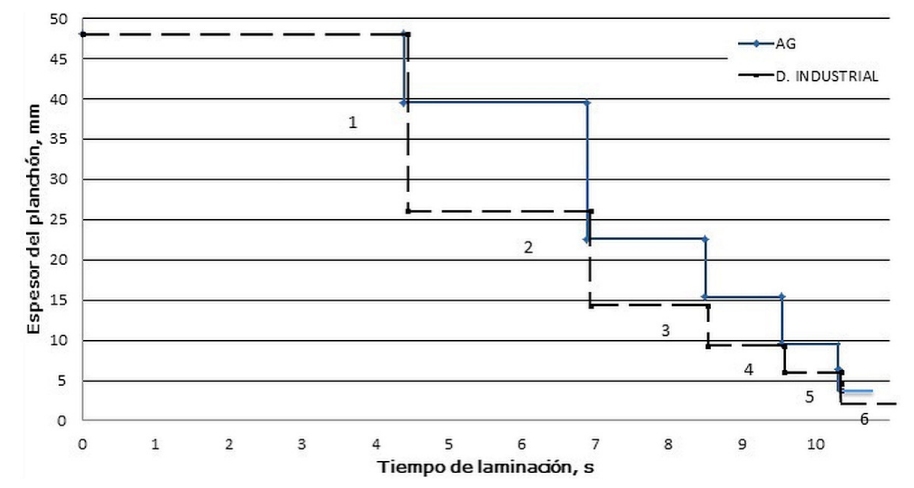

Fig. 6. Comparación entre la cédula obtenida por el algoritmo genético y la cédula industrial. Los números indican el paso de laminación.

\section{Conclusiones}

- En este trabajo se implementó el NSGA-II para determinar un conjunto de 100 soluciones a un bajo costo computacional.

- La implementación del NSGA-II con sus variantes AG1 y AG2 minimizaron la flexión de los rodillos de trabajo con respecto a la obtenida en datos industriales y mejoró en un $0.37 \%$ el tiempo de laminación industrial.

- La minimización de la corona de flexión asegura que la planicidad de la lámina siempre siempre fue menor a la establecida en normas internacionales.

- Se demostró la factibilidad de la aplicación de algoritmos genéticos multiobjetivo a esta clase de problemas por su fácil adaptación a modelos matemáticos complejos, así como al bajo costo computacional.

Agradecimientos. Este trabajo fue realizado con fondos del Instituto Politécnico Nacional (proyectos clave SIP201612163 y 20161930). Se agradece a Richard Shulkosky de la compañía INTEG Process Group por proporcionar una versión demo del simulador HSMM.

\section{Referencias}

1. Petty, D.M.: Application of process modelling-an industrial view. J Mater Proc Technol 60, pp. 421-426 (1996)

2. Tseng, A.A., Tong, S.X., Chen, T.C.: Thermal expansion and crown evaluations in rolling processes. Mater Design 17, pp. 193-204 (1996)

3. Nolle, L., Armstrong, A., Hopgood, A., Ware, A.: Optimum Work Roll Profile Selection in the Hot Rolling of Wide Steel Strip Using Computational Intelligence. In: Computational Intelligence: Theory and Applications International Conference, Dortmund, Germany, Reusch, B. (eds). Springer, Berlin (1999) 
4. Holland, J.H.: The dynamics of searchers directed by genetic algorithms. En Lee, Y.C. (Ed.), Evolution, learning, and cognition, Teaneck, N. J. World Scientific (1988)

5. Zitzler, E., Deb, K., Thiele, L.: Comparison of multiobjective evolutionary algorithms: empirical results. Evol Comput 8, pp. 173-195 (2000)

6. Deb, K.: Multi-Objective Optimization Using Evolutionary Algorithms: An Introduction. pp. 209-218, Wiley, New York (2001)

7. Wang, D.D., Tieu, A.K., de Boer, F.G., Ma, B., Yuen, W.Y.D.: Toward a heuristic optimum design of rolling schedules for tandem cold rolling mills. Eng Appl Artificial Intell 13(4), pp. 397-406 (2000)

8. Nolle, L., Zelinka, I.: SOMA applied to Optimum Work Roll Profile Selection in the hot rolling of wide steel. In: 17th European Simulation Multiconference, Nottingham, UK (2003)

9. Nolle, L.: SASS applied to optimum work roll profile selection in the hot rolling of wide steel. Knowledge-Based Systems 20(2), pp. 203-208 (2007)

10. Pholdee, N., Bureerat, S., Park, W.W., Kim, D.K., Im, Y.T., Kwon, H.C., Chun, M.S.: Optimization of Flatness of Strip during Coiling Process based on Evolutionary Algorithms. Int J Precis Eng Man 16(7), pp. 1493-1499 (2015)

11. Hernández Carreón, C.A., Fraire Huacuja, H.J., Espriella Fernandez, K., Valdez Castilla, G., Mancilla Tolama, J.E.: Application of Genetic Algorithms to Strip Hot Rolling Scheduling. In: Corchado, E. y col. (eds.) Innovations in Hybrid Intelligent Systems, vol. 44. Advances in Soft Computing, pp. 247-254. Springer, Berlin (2007)

12. Hernández Carreón, C.A., Fraire-Huacuja, H.J., Fernandez, K.E., Castilla-Valdez, G., Mancilla Tolama, J.E.: A Model to Minimize the Hot Rolling Time of a Steel Slab Considering the Steel Chemical Composition. In: Corchado, J.M. y col. (eds.) International Symposium on Distributed Computing and Artificial Intelligence 2008, vol. 50. Advances in Soft Computing, pp. 471-480. Springer, Berlin (2009)

13. Espriella, K: Optimización mediante algoritmos genéticos: aplicación a la laminación en caliente. Tesis de Maestría, Instituto Politécnico Nacional, CICATA Unidad Altamira (2008) 